\title{
Editorial Introduction Special Issue on Memetic Algorithms
}

It is now 15 years since Moscato recognised a growing trend, and first coined the term "Memetic Algorithms" to refer to the combination of Evolutionary Algorithms (EAs) with Local Search or other improvement techniques for refining solutions. Since that time Memetic Algorithms (MAs) have been successfully applied to hundreds of real-world problems and have been the subject of intense scientific research both in academia and industry. The implementation of ever more sophisticated MAs has been made possible thanks to advances in computing capabilities. Consequently their use has spread to domains that range from the construction of optimal university exam timetables, to the prediction of protein structures and the optimal design of space-craft trajectories.

The importance of Memetic Algorithms in both real-world applications and academic research has lead to the establishment of the series of international Workshops On Memetic Algorithms (WOMA) and a dedicated book. WOMA has served as a forum for the exchange of ideas and knowledge on Memetic Algorithms and the fifth workshop in the series will take place in Birmingham, UK, within the auspices of PPSN. From these workshops, and other research, three principal threads of discussion have emerged.

- Integration: It is clear that there are many different ways in which the local search or improvement heuristics may be combined with the EA. It seems equally clear that some of these may lead to performance benefits through a synergistic globallocal search trade-off, but that this is not guaranteed. The question of when to apply local improvement heuristics, to which individuals in EAs population and how much computational effort to devote to them remains unanswered, and more research effort is required to gain the understanding and insights that may lead to guidelines for the design of efficient and effective algorithms.

- Analysis: Currently the theoretical analysis of MAs lags some way behind that of "pure" EAs. Given the plethora of methods now available, it remains to be seen which tools, techniques and approaches will be useful for gaining insight into MA behaviour.

- Exploitation: Successful applications have been the driving force behind much of the research in this area. Although these case studies demonstrate the potential utility of MAs, they also serve to illustrate how problem-specific information can be exploited to adapt this meta-heuristic to maximize its performance. In MA research, as in other research in the broader EA field, there is a tendency to rely on established and well-known test functions, sets, or problem generators. The study of the algorithmic details necessary to get state-of-the-art performance on real-world problems can be of help in avoiding some of the possible artifacts induced by academic benchmarks.

The papers in the special issue represent a broad spectrum of MA research that spans these three issues. 
The paper by Lozano et al. describes the development and testing of a new Memetic Algorithm for real-coded problems. This algorithm probabilistically applies a new crossover-based hill-climbing method within a steady-state EA. The probability of applying local search is fitness-based, and is shown to permit the adaptation of the ratio of time spent on local or global search to the problem in hand. Moreover the authors specifically address one of the key issues in the design of effective MAs, namely how to avoid the tendency of local search to accelerate the loss of diversity.

The paper by Merz concerns the utility of various landscape metrics to aid in understanding the behaviour of a particular class of MAs which has been proven to be competitive on a number of combinatorial optimisation problems. These algorithms apply Lamarkian local search to every offspring, so that at any stage the EA is working with a subset of solutions which are locally optimal with respect to some move operator. This renders many of the previously used landscape metrics inapplicable, and the author describes several new metrics which may be of use in characterising the interplay between algorithm and problem instance, and ultimately in developing efficient adaptive MAs.

The paper by Areibi and Yang details the development of a MA for two problems in VLSI design. Not only does this provide a useful "success story", but it also examines several different ways in which EAs may be combined with other heuristics so as to maximise the exploitation of prior knowledge and expertise. Thus the underlying EA is augmented with not only a powerful problem-specific local search method, but also with a greedy constructive heuristic for creating a high-quality initial population, and an adaptive problem decomposition heuristic. The analysis shows some interesting synergies between these different heuristics.

We would like to take this opportunity to thank all those authors who submitted papers, and all of the reviewers who took such care in reviewing these papers. We hope that the selected papers will serve not only as a reflection of the current state of the art in MAs, but also as a inspiration and spur to other researchers.

W.E. Hart, N. Krasnogor and J.E. Smith, Guest Editors

June 4. 2004

\section{References}

Hart, W.E., Krasnogor, N., and Smith, J.E., editors (2004). Recent Advances in Memetic Algorithms, Springer, Berlin, Heidelberg, New York.

Moscato, P.A. (1989). On Evolution, Search, Optimization, Genetic Algorithms and Martial Arts: Towards Memetic Algorithms. Caltech Concurrent Computation Program Report 826, Caltech, Pasadena, California.

Krasnogor, N. (2004). Schedules and presented papers at WOMA I-IV. Available from http://www.cs.nott.ac.uk/ñxk/ 